\title{
Risk Factors of Transition from Normal Cognition to Mild Cognitive Disorder: The PATH through Life Study
}

\author{
Nicolas Cherbuin ${ }^{a}$ Chantal Reglade-Meslin ${ }^{a}$ Rajeev Kumar ${ }^{a}$ b Patricia Jacomb ${ }^{a}$ \\ Simon Eastealc Helen Christensen ${ }^{a}$ Perminder Sachdev ${ }^{d, e}$ Kaarin J. Anstey ${ }^{a}$ \\ ${ }^{a}$ Centre for Mental Health Research, ${ }^{b}$ Department of Psychological Medicine, and ' John Curtin School of Medical \\ Research, Australian National University, Canberra, A.C.T., and d School of Psychiatry, University of New South Wales, \\ and ${ }^{\mathrm{e}}$ Neuropsychiatric Institute, Sydney, N.S.W., Australia
}

\section{Key Words}

Mild cognitive impairment - Alcohol $\cdot$ Hypertension - BMI • Depression

\begin{abstract}
Aims: The aim of this study was to identify physical and mental health and lifestyle predictors of transition from normal cognition to mild cognitive disorder (MCD). Methods: A total of 2,082 individuals, aged 60-64 years, were assessed at 2 time-points 4 years apart for mild cognitive impairment (MCl) and other MCDs. Results: The main predictors of conversion to $\mathrm{MCl}$ and to other mild cognitive disorders were past alcohol intake, current anxiety and depression medication, increased systolic blood pressure, and past smoking. Conclusion: Participants with a history of smoking or harmful alcohol consumption, hypertension, or who took medication for anxiety or depression were at increased risk of transitioning to $\mathrm{MCl}$ or any MCD. Strategies targeted at managing the above risk factors may have benefits in preventing mild cognitive decline in relatively healthy middle-aged individuals living in the community.

Copyright ๑ 2009 S. Karger AG, Basel
\end{abstract}

\section{Introduction}

There is considerable evidence for an association between mild cognitive disorders (MCDs) in ageing and Alzheimer's disease (AD) [1], with many studies showing high rates $(\sim 30 \%)$ of conversion from MCD to $\mathrm{AD}$ over relatively short periods of time ( $2-5$ years) [2]. However, few studies have reported on factors predicting transition from normal cognitive ageing to clinically assessed MCD or on factors protecting individuals from such a transition, and those who did generally assessed older individuals [3]. Based on post-mortem studies, Braak et al. [4] have shown that the neurodegenerative processes leading to AD start early in adulthood and progress over decades. It may follow that known risk factors for conversion to dementia are also predictive of conversion to MCD and, therefore, might be amenable to even earlier intervention.

With the aim of identifying these factors in younger individuals, we investigated a community-based cohort of 60-64-year-old participants taking part in a large prospective longitudinal study of ageing. We expected, aside from age, cardiovascular disease [5], diabetes [6], obesity [7], head injury [8], hypercholesterolemia [9], excessive alcohol consumption [10], depression [11], smoking [12],

Tel. +61 26125 3858, Fax +61 26125 0733,E-Mail nicolas.cherbuin@anu.edu.au 
poor visual acuity [13], and $\mathrm{APOE}^{*} \mathrm{E} 4$ genotype [14] to increase the risk, and moderate alcohol consumption, physical fitness (forced expiratory and vital capacity), handgrip, mental well-being, and $\mathrm{APOE}^{*} \mathrm{E} 2$ genotype to be protective factors.

One problem in such research relates to the lack of a single universally acceptable definition of MCD as a prodrome to AD. While the MCI definition proposed by the International Consensus Conference is widely used, the concept is not very stable especially in individuals in their 60 s, with a large proportion of those assessed reverting to a non-MCI status at follow-up $[15,16]$. In contrast, we have shown the stability of the classification with any mild cognitive disorders, i.e. any of Age Associated Memory Impairment (AAMI), Age Associated Cognitive Decline (AACD), Mild Neurocognitive Disorder (MNC) or $\mathrm{MCI}$, to be high ( $\sim 89 \%)$ [16]. For this reason, we investigated the predictors for both MCI and any MCD (any$\mathrm{MCD}$ ) in the present study.

\section{Methods}

\section{Study Population}

The design of the PATH Through Life Study has been described elsewhere [17] as has the clinical sub-study [18]. Briefly, participants who were residents of the city of Canberra and the adjacent town of Queanbeyan, Australia, were recruited randomly through the electoral roll to participate in a study interested in the risk and protective factors for normal ageing, dementia and other neuropsychiatric disorders. Enrolment to vote is compulsory for Australian citizens. Participants were recruited in the 3 age cohorts 20-24, 40-44, and 60-64 and are to be followed every 4 years, over a total period of 20 years. The study was approved by the Australian National University Ethics Committee. Results presented here concern the first- and second-wave interviews with 60-64-year-olds which were conducted in 2001-2002 and 2005-2006. Of 4,831 people contacted, 2,551 (58.3\%) were interviewed in wave 1 . Of these, 2,222 individuals completed wave 2 assessments. A further 139 participants were excluded from analysis because they were either diagnosed with a cognitive disorder at wave $1(n=75)$ or refused dementia assessment after screening positive on cognitive impairment measures $(n=64)$. One participant was excluded due to the loss of wave 1 measures (fig. 1).

\section{Clinical Assessment}

The screening procedures and clinical diagnosis for wave 1 and wave 2 have been described previously $[9,16,18]$. In brief, diagnoses were obtained for MCI [19], AAMI [20], AACD [21], MNC [22], impairment on the clinical dementia rating $(\mathrm{CDR}=$ $0.5)$ [23], and other cognitive disorder (OCD) using published criteria [24]. DSM-IV criteria [22] were used to assess dementia and the same assessment procedure was used at wave 1 and 2 .

As we have previously reported high stability of diagnosis of any mild cognitive disorder (i.e. MNC, OCD, AAMI, MCI, AACD)

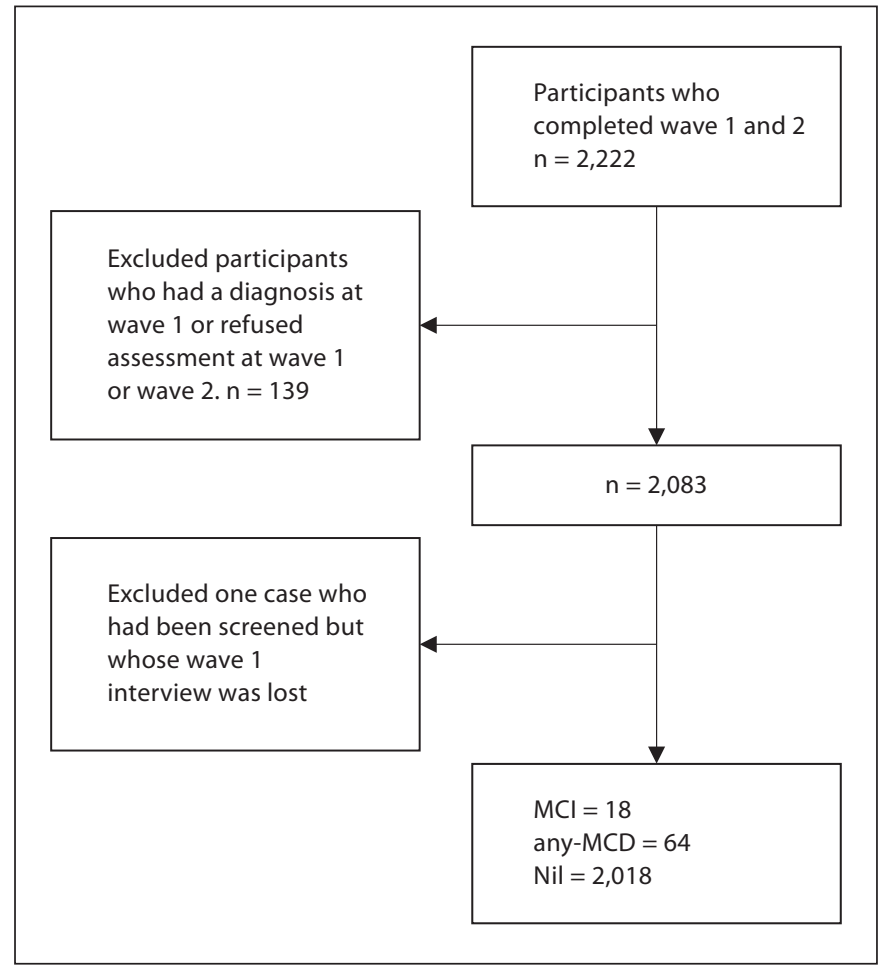

Fig. 1. Sample selection.

over a 4-year follow-up [16], participants were also classified with any-MCD and analyses were conducted to identify predictors of this more general classification.

\section{Physical Health Measures}

Diastolic and systolic blood pressure (continuous measures) were measured twice while seated and after a rest period during the interview. Self-reported diabetes and diabetes medication were recorded (no $=0 /$ yes $=1$ ). Forced vital capacity (FVC, continuous) was measured using the forced expiratory vital capacity maneuver with a spirometer (Micro Spirometer, Micro Medical Ltd., Rochester, UK) using the best of 3 attempts. Physical health was measured with the SF-12, a validated 12 -item self-administered inventory based on which physical and mental health components can be computed with higher scores reflecting better health [25]. Self-reported measures of weight and height were used to compute a body mass index (BMI, continuous) measure. Stroke, and arthritis were assessed by self-report (no $=0 /$ yes $=1$ ). APOE status was assessed by buccal swabs and is described elsewhere [26].

\section{Mental Health Measures}

Self-rated mental health was measured by the component summary of the SF-12, with higher scores reflecting better health [25]. Anxiety and depression symptoms in the past month were assessed by the Goldberg anxiety/depression scales which consist of an 18-item self-report symptom inventory with higher scores reflecting more anxiety/depression symptoms [27]. 


\section{Medications}

Information on type and number of current medications was recorded. Medication for hypertension, diabetes, hypercholesterolemia, anxiety, and depression (no $=0$ /yes $=1$ ) were examined, as these conditions have previously been associated with cognitive decline [28].

\section{Lifestyle Measures}

Alcohol consumption was assessed using the Alcohol Use Disorders Identification Test [AUDIT; 29]. Categorical estimates of weekly consumption were derived from the quantity and frequency items of the AUDIT. For men, weekly alcohol consumption was categorized from 1-6 as abstainer, light (1-13 units), moderate (14-27 units), hazardous (28-42 units), or harmful ( $>42$ units) (i.e. 1 = abstainer; 6 = harmful). For women, weekly alcohol consumption was divided into abstainer, light (1-7 units), moderate (8-13 units), hazardous (14-28 units) or harmful ( $>28$ units) categories where a unit equates $10 \mathrm{~g}$ of pure alcohol. Weekly consumption of standard drinks was estimated from items measuring reported frequency of alcohol intake, and the number of standard drinks consumed on typical drinking days, following quantity-frequency assessment procedures. Current and past smoking habits were recorded (no $=0 /$ yes $=1$ ).

\section{Statistical Analysis}

Group differences were assessed using $\chi^{2}$ tests for categorical variables and t tests for continuous variables. Logistic regression analysis was used to identify significant predictors of binary group membership (normal vs. MCI/any-MCD) using a stepwise approach. In the first phase, each health and lifestyle variable was assessed in a univariate analysis while controlling for age, sex, and education. In the second phase, multivariate logistic regression analyses were conducted to determine which variables had the highest predictive value. All variables were entered together and the model was progressively reduced until it only included significant predictors after controlling for age, sex, and education. The alpha level was set at $\mathrm{p}=0.05$. Sex interactions were investigated.

\section{Results}

Table 1 presents the demographic, clinical, and genetic characteristics of the study groups (normal, MCI, and any-MCD). Eighteen MCI and 64 any-MCD (18 MCI, 24 AAMI, 14 MNC, 3 OCD, and 63 impaired on CDR) individuals were identified from the 2,082 participants with no diagnosis at wave 1 . No case of dementia was identified.

\section{Predictors of Conversion to MCI}

FVC (odds ratio (OR) 0.45; 95\% confidence interval (CI) $0.44-0.46 ; \mathrm{p}=0.029$ ), anxiety medication (OR 4.08; 95\% CI 1.16-14.39; $\mathrm{p}=0.029)$, anti-depressive medication (OR 4.12; 95\% CI 1.16-14.64; $\mathrm{p}=0.029$ ), and alcohol abstinence (OR 9.10; 95\% CI 3.02-27.42; p <0.001) were sig- nificant predictors of conversion to MCI identified by univariate logistic analyses while controlling for sex, age, and education (table 2). When all variables were assessed in a single multivariate analysis, and after reduction of the model, past alcohol intake (OR 0.59; 95\% CI 0.37$0.92 ; \mathrm{p}=0.021$ ), quadratic trend for past alcohol intake (modelling a U-shape and indicating a higher risk for low and high drinking categories: OR 1.58; 95\% CI 1.18-2.11; $\mathrm{p}=0.002$ ), and past smoking (OR 3.22; 95\% CI 1.05-9.87; $\mathrm{p}=0.041)$ were significant predictors of conversion to MCI (table 3). Trends were also detected for anxiety medication (OR 3.58; 95\% CI 0.97-13.22; p =0.056), visual acuity (OR 0.92; 95\% CI 0.85-1.00; p = 0.059), and $\mathrm{APOE}^{*} \mathrm{E} 2$ genotype (OR 2.74; 95\% CI 0.95-7.97; $\mathrm{p}=$ 0.063). $\mathrm{APOE}^{*} \mathrm{E} 4 \mathrm{APOE}$ genotype was not a significant predictor of conversion to MCI. None of the sex interactions reached significance.

\section{Predictors of Conversion to Any-MCD}

Taking antidepressant medication (OR 3.71; 95\% CI $1.76-7.81 ; \mathrm{p}=0.001)$, past smoking (OR 1.78; 95\% CI 1.06$3.00 ; \mathrm{p}=0.031$ ), arthritis (OR 1.73; 95\% CI 1.04-2.90; $\mathrm{p}=$ 0.037 ), depression (OR 1.15; 95\% CI 1.02-1.29; $\mathrm{p}=0.026$ ), BMI (OR 1.05; 95\% CI 1.05-1.09; p = 0.025), physical health (SF-12, OR 0.97; 95\% CI 0.95-0.99; p =0.011), taking cholesterol-lowering medication (OR 2.01; 95\% CI 1.20-3.37; $\mathrm{p}=0.008$ ) and oral diabetes medication (OR 2.87 ; 95\% CI 1.26-6.53; $\mathrm{p}=0.012$ ) were significant predictors of conversion to any-MCD identified by univariate logistic analyses while controlling for sex, age, and education (table 2). When all variables were assessed in a single multivariate analysis, and after reduction of the model, diastolic blood pressure (OR 0.96; 95\% CI 0.92-0.99; $\mathrm{p}=$ 0.011), past smoking (OR 1.97; 95\% CI 1.12-3.44; $\mathrm{p}=$ 0.018), taking depression medication (OR 3.25; 95\% CI $1.51-7.00 ; \mathrm{p}=0.003)$ and past alcohol intake category $(\mathrm{OR}$ $0.75 ; 95 \%$ CI $0.57-1.00 ; \mathrm{p}=0.046)$ were significant predictors of conversion to MCI (table 4). Trends were also detected for BMI (OR 1.04; 95\% CI 1.00-1.08; p = 0.054), systolic blood pressure (OR 1.02; 95\% CI 1.00-1.04; p = 0.057 ), and a quadratic trend for past alcohol intake (modelling a U-shape and indicating a higher risk for low and high drinking categories: OR 1.17; 95\% CI 0.98-1.40; $\mathrm{p}=$ 0.087). Neither ${ }^{*} \mathrm{E} 4$ nor ${ }^{*} \mathrm{E} 2 \mathrm{APOE}$ genotype were significant predictors of conversion to MCI. None of the sex interactions reached significance. 
Table 1. Demographic, clinical, and genetic characteristics of normal, MCI, and any-MCD sub-samples at baseline (wave 1)

\begin{tabular}{|c|c|c|c|}
\hline & $\begin{array}{l}\text { Normal at wave } 2 \\
(\mathrm{n}=2,018)\end{array}$ & $\begin{array}{l}\text { MCI at wave } 2 \\
(\mathrm{n}=18)\end{array}$ & $\begin{array}{l}\text { Any-MCD at wave } 2 \\
(\mathrm{n}=64)\end{array}$ \\
\hline Females, $\mathrm{n}$ & $996(49.2 \%)$ & $8(44.4 \%)$ & $24(40.7 \%)$ \\
\hline Age at wave 1 , years & $62.53 \pm 1.51$ & $62.22 \pm 1.77$ & $62.53 \pm 1.63$ \\
\hline Education, years & $14.05 \pm 2.67$ & $13.11 \pm 2.15$ & $13.13 \pm 2.36^{* *}$ \\
\hline Caucasian & $1,850(96.60 \%)$ & $15(83.30 \%)^{*}$ & $60(93.80 \%)$ \\
\hline English-speaking & $1,822(90.30 \%)$ & $12(66.70 \%)^{* *}$ & $54(84.40 \%)$ \\
\hline \multicolumn{4}{|l|}{ MMSE } \\
\hline At wave 1 & $29.37 \pm 0.895$ & $29.11 \pm 0.83^{* *}$ & $28.92 \pm 0.981^{* *}$ \\
\hline At wave 2 & $29.33 \pm 0.96$ & $27.5 \pm 1.65^{* *}$ & $27.94 \pm 1.84^{* *}$ \\
\hline $\mathrm{APOE}^{*} \mathrm{E} 4$ carriers & $526(26.06 \%)$ & $3(16.70 \%)$ & $12(18.75 \%)$ \\
\hline $\mathrm{APOE}^{*} \mathrm{E} 2$ carriers & $278(13.78 \%)$ & $5(27.78 \%)$ & $11(17.20 \%)$ \\
\hline Diabetes & $135(6.70 \%)$ & $1(5.60 \%)$ & $9(14.10 \%)^{*}$ \\
\hline Taking oral diabetes medication & $77(3.80 \%)$ & $1(5.60 \%)$ & $7(10.90 \%)^{*}$ \\
\hline Taking cholesterol-lowering medication & $467(23.10 \%)$ & $4(22.20 \%)$ & $25(39.10 \%)^{* *}$ \\
\hline Taking blood pressure medication & $633(31.40 \%)$ & $7(38.90 \%)$ & $28(43.80 \%)^{*}$ \\
\hline Stroke & $72(3.60 \%)$ & $0(0.00 \%)$ & $5(7.80 \%)$ \\
\hline Head injury & $107(5.30 \%)$ & $0(0.00 \%)$ & $6(9.40 \%)$ \\
\hline Arthritis & $682(33.80 \%)$ & $9(50.00 \%)$ & $30(46.90 \%)^{*}$ \\
\hline Taking anxiety medication & $93(4.60 \%)$ & $3(16.70 \%)^{*}$ & $5(7.80 \%)$ \\
\hline Taking depression medication & $93(4.60 \%)$ & $3(16.70 \%)^{*}$ & $9(14.10 \%)^{* *}$ \\
\hline Taking memory medication & $59(2.90 \%)$ & $1(5.60 \%)$ & $2(3.10 \%)$ \\
\hline Goldberg depression scale & $1.54 \pm 1.79$ & $2.08 \pm 1.98$ & $2.28 \pm 2.30^{*}$ \\
\hline Goldberg anxiety scale & $2.14 \pm 2.24$ & $2.33 \pm 2.34$ & $2.06 \pm 2.36$ \\
\hline \multicolumn{4}{|l|}{ Past alcohol intake categories } \\
\hline Abstainer & $93(4.60 \%)$ & $5(27.80 \%)^{* *}$ & $6(9.40 \%)$ \\
\hline Occasional & $462(22.90 \%)$ & $3(16.70 \%)$ & $18(28.10 \%)$ \\
\hline Light & $674(33.40 \%)$ & $4(22.20 \%)$ & $19(29.70 \%)$ \\
\hline Moderate & $435(21.60 \%)$ & $1(5.60 \%)$ & $8(12.50 \%)$ \\
\hline Hazardous & $224(11.10 \%)$ & $4(22.20 \%)$ & $8(12.50 \%)$ \\
\hline Harmful & $130(6.40 \%)$ & $1(5.60 \%)$ & $5(7.80 \%)$ \\
\hline Past smoker & $909(44.00 \%)$ & $11(61.10 \%)$ & $38(59.40 \%)^{*}$ \\
\hline Present smoker & $192(9.30 \%)$ & $0(0.00 \%)$ & $7(10.90 \%)$ \\
\hline Systolic blood pressure, $\mathrm{mm} \mathrm{Hg}$ & $140 \pm 18.71$ & $140.89 \pm 17.30$ & $140.77 \pm 24.08$ \\
\hline Diastolic blood pressure, $\mathrm{mm} \mathrm{Hg}$ & $83 \pm 10.51$ & $81.08 \pm 8.44$ & $81.18 \pm 12.09$ \\
\hline Forced vital capacity, 1 & $3.11 \pm 0.84$ & $2.81 \pm 0.65$ & $2.99 \pm 0.82$ \\
\hline BMI & $26.79 \pm 4.93$ & $27.35 \pm 4.00$ & $28.35 \pm 6.84$ \\
\hline Physical health self-report (SF-12) & $72.36 \pm 27.46$ & $72.82 \pm 30.09$ & $65.48 \pm 29.23^{*}$ \\
\hline Mental health self-report (SF-12) & $81.49 \pm 29.41$ & $78.14 \pm 30.60$ & $78.27 \pm 29.63$ \\
\hline Visual acuity (no. of letters on Snellen chart) & $25.86 \pm 3.89$ & $25.05 \pm 4.58$ & $24.06 \pm 5.88$ \\
\hline
\end{tabular}

${ }^{*} \mathrm{p}<0.05,{ }^{* *} \mathrm{p}<0.01$ compared with normal sample.

\section{Discussion}

The main strengths of this study are the investigation of a wide range of health and lifestyle risk factors for MCI in a large and comparatively young community-based cohort of older adults. In addition, predictors of a less specific clinical group, having any MCD, were also investigated because we have previously shown [16] that the any-MCD is more stable than an MCI diagnosis based on consensus criteria. That is, of those diagnosed with MCI or any-MCD, a large proportion (71\%) of those diagnosed with MCI reverted to non-MCI status 4 years later, whereas only $11 \%$ of any-MCD individuals lost their diagnosis (there were no dementia diagnoses). Consequently, the any-MCD is seen as a clinical category overlapping and sharing many characteristics with MCI. The differences in predictors discussed below are therefore thought to predominantly indicate difference in statistical power af- 
Table 2. Health and lifestyle predictors of transition from normal ageing to MCI and any-MCD adjusted for age, sex and education

\begin{tabular}{|c|c|c|c|c|}
\hline \multirow[t]{2}{*}{ Predictors } & \multicolumn{2}{|l|}{ MCI } & \multicolumn{2}{|l|}{ Any-MCD } \\
\hline & Odds ratio $(95 \% \mathrm{CI})$ & $\mathrm{p}$ & Odds ratio $(95 \% \mathrm{CI})$ & $\mathrm{p}$ \\
\hline Diabetes & $0.73(0.95-5.52)$ & 0.756 & $2.06(0.99-4.28)$ & 0.054 \\
\hline Taking oral diabetes medication & $1.30(0.17-9.96)$ & 0.799 & $2.87(1.26-6.53)$ & 0.012 \\
\hline Taking cholesterol-lowering medication & $0.88(0.29-2.70)$ & 0.822 & $2.01(1.20-3.37)$ & 0.008 \\
\hline Taking blood pressure medication & $1.33(0.51-3.45)$ & 0.563 & $1.64(0.99-2.71)$ & 0.056 \\
\hline Systolic blood pressure, mm Hg & $1.00(0.98-1.03)$ & 0.878 & $1.00(0.99-1.02)$ & 0.838 \\
\hline Diastolic blood pressure, $\mathrm{mm} \mathrm{Hg}$ & $0.98(0.93-1.02)$ & 0.332 & $0.98(0.95-1.03)$ & 0.080 \\
\hline Stroke & - & - & $2.33(0.91-6.02)$ & 0.079 \\
\hline Arthritis & $1.97(0.76-5.12)$ & 0.163 & $1.73(1.04-2.90)$ & 0.037 \\
\hline FVC, 1 & $0.45(0.44-0.46)$ & 0.029 & $0.69(0.47-1.03)$ & 0.068 \\
\hline BMI & $1.01(0.93-1.10)$ & 0.752 & $1.05(1.05-1.09)$ & 0.025 \\
\hline Physical health self-report & $1.02(0.97-1.09)$ & 0.359 & $0.97(0.95-0.99)$ & 0.011 \\
\hline Taking anxiety medication & $4.08(1.16-14.39)$ & 0.029 & $1.76(0.69-4.51)$ & 0.238 \\
\hline Goldberg anxiety scale & $0.98(0.79-1.21)$ & 0.856 & $1.04(0.93-1.15)$ & 0.521 \\
\hline Taking depression medication & $4.12(1.16-14.64)$ & 0.029 & $3.71(1.76-7.81)$ & 0.001 \\
\hline Goldberg depression scale & $1.19(0.96-1.46)$ & 0.106 & $1.15(1.02-1.29)$ & 0.026 \\
\hline Mental health self-report & $0.99(0.99-1.05)$ & 0.705 & $1.00(0.96-1.03)$ & 0.823 \\
\hline Past alcohol intake category ( $1=$ abstainer; $6=$ harmful $)$ & $0.76(0.50-1.15)$ & 0.200 & $0.88(0.72-1.09)$ & 0.253 \\
\hline Past alcohol intake (quadratic trend) & $1.48(1.13-1.94)$ & 0.005 & $1.16(0.97-1.38)$ & 0.097 \\
\hline Abstain to drink alcohol (vs. all others) & $9.10(3.02-27.42)$ & $<0.001$ & $2.23(0.92-5.39)$ & 0.076 \\
\hline Harmful/hazardous past drinking (vs. all others) & $1.76(0.61-5.14)$ & 0.298 & $1.15(0.61-2.17)$ & 0.671 \\
\hline Past smoker & $1.88(0.71-5.02)$ & 0.203 & $1.78(1.06-3.00)$ & 0.031 \\
\hline Visual acuity (Snellen) & $0.93(0.86-1.01)$ & 0.073 & $0.96(0.91-1.01)$ & 0.139 \\
\hline $\mathrm{APOE}^{*} \mathrm{E} 4$ & $0.55(0.16-1.93)$ & 0.354 & $0.66(0.35-1.24)$ & 0.193 \\
\hline $\mathrm{APOE}^{*} \mathrm{E} 2$ & $2.45(0.86-6.93)$ & 0.092 & $1.31(0.67-2.54)$ & 0.427 \\
\hline
\end{tabular}

forded by different sample sizes rather than instrumental differences supported by fundamentally different underlying pathological processes.

As predicted, past smoking, and taking anxiety/depression medications were associated with increased risk of MCI and any-MCD in all analyses while alcohol consumption was found to be protective (when not taken in hazardous quantities) after controlling for sex, age, and education. These factors have been identified in previous research investigating conversion to dementia [30], but the fact that they can be reliably detected in a relatively young sample known to be cognitively intact at the start of the study and in relation to MCI and any-MCD, not dementia, is important because it confirms the relevance of $\mathrm{MCI}$ as a precursor stage to dementia and suggests a progressive effect of certain risk and protective factors for dementia.

Past smoking, which has been consistently associated with increased risks of developing dementia [see 12 for a review] was associated with a 2-3-fold increase in risk of developing MCI and any-MCD. The mechanisms mediating this effect are not fully understood but include an increased risk of cardiovascular disease in smokers. Recent research in rodents [31] has shown that exposure to cigarette smoke promotes arterial thrombosis and increases arterial wall thickening which are likely to lead to decreased perfusion, increased risk of stroke, and increased blood pressure. White matter hyperintensities, which are thought to reflect small vessel disease, are associated with hypertension and cardiovascular disease and are a risk factor for dementia. An association between smoking and white matter hyperintensities has been demonstrated and is consistent with a cardiovascular effect of smoking on cognition. These findings strengthen the view that smoking is bad for mental health and should be avoided.

Alcohol consumption was assessed using the AUDIT, which has been shown to have good psychometric characteristics in a variety of contexts and cultures [e.g. 32]. The association between alcohol consumption and cognitive impairment was complex and followed both linear and quadratic functions, with abstinence and high intake being associated with increased risk and moderate consumption being associated with decreased risk of both 
Table 3. Health and lifestyle predictors of conversion to MCI (full and reduced models)

\begin{tabular}{|c|c|c|c|c|}
\hline \multirow[t]{2}{*}{ Models } & \multicolumn{2}{|l|}{ Full } & \multicolumn{2}{|c|}{ Reduced } \\
\hline & Sig. & $\operatorname{Exp}(B)$ & Sig. & $\operatorname{Exp}(B)$ \\
\hline Systolic blood pressure, $\mathrm{mm} \mathrm{Hg}$ & 0.360 & $1.02(0.98-1.05)$ & & \\
\hline Diastolic blood pressure, $\mathrm{mm} \mathrm{Hg}$ & 0.128 & $0.95(0.88-1.02)$ & & \\
\hline Arthritis & 0.238 & $1.89(0.66-5.46)$ & & \\
\hline FVC, 1 & 0.098 & $0.52(0.24-1.13)$ & & \\
\hline Diabetes & 0.563 & $0.53(0.06-4.6)$ & & \\
\hline BMI & 0.882 & $1.01(0.91-1.11)$ & & \\
\hline Physical health self-report & 0.033 & $1.08(1.01-1.15)$ & & \\
\hline Mental health self-report & 0.309 & $1.05(0.95-1.16)$ & & \\
\hline Visual acuity (Snellen) & 0.051 & $0.92(0.84-1)$ & 0.059 & $0.92(0.85-1.00)$ \\
\hline Taking blood pressure medication & 0.547 & $1.41(0.46-4.3)$ & & \\
\hline Taking anxiety medication & 0.317 & $2.6(0.4-16.9)$ & 0.056 & $3.58(0.97-13.22)$ \\
\hline Taking depression medication & 0.314 & $2.79(0.38-20.57)$ & & \\
\hline Taking cholesterol-lowering medication & 0.436 & $0.6(0.16-2.18)$ & & \\
\hline Goldberg anxiety scale & 0.293 & $0.83(0.58-1.18)$ & & \\
\hline Goldberg depression scale & 0.021 & $1.54(1.07-2.22)$ & & \\
\hline Past smoker & 0.050 & $3.26(1-10.67)$ & 0.041 & $3.22(1.05-9.87)$ \\
\hline Past alcohol intake category & 0.031 & $0.58(0.36-0.95)$ & 0.021 & $0.59(0.37-0.92)$ \\
\hline Past alcohol intake category (quadratic) & 0.009 & $1.52(1.11-2.08)$ & 0.002 & $1.58(1.18-2.11)$ \\
\hline $\mathrm{APOE}^{*} \mathrm{E} 4$ & 0.458 & $0.61(0.16-2.26)$ & & \\
\hline $\mathrm{APOE}^{*} \mathrm{E} 2$ & 0.089 & $2.67(0.86-8.27)$ & 0.063 & $2.74(0.95-7.97)$ \\
\hline \multirow[t]{2}{*}{ Model fit } & $\mathrm{R}^{2}$ & -2 Log likelihood & $\mathrm{R}^{2}$ & -2 Log likelihood \\
\hline & 0.218 & 163.52 & 0.130 & 181.20 \\
\hline
\end{tabular}

All models are adjusted for age, sex, and education.

MCI and any-MCD. This is consistent with previous research showing that moderate alcohol consumption is protective of dementia [33] and with cross-sectional findings of the PATH study showing an inverse $\mathrm{U}$-association between drinks per week and cognitive performance [34].

Depression and anxiety are known risk factors for dementia, but it remains unclear whether they occur in the prodrome to dementia or are causally related. Recently, Palmer et al. [30] showed that individuals with both MCI and anxiety symptoms were more than 13 times more likely to develop AD than cognitively intact individuals and twice as likely compared to individual with MCI without anxiety symptoms [30]. In the present study, participants taking anxiety or depression medication had a 4 -fold increase in risk of developing MCI or any-MCD. Self-reported anxiety or depression, however, was not or only mildly predictive.

Cardiovascular measures were significant predictors of transition to any-MCD, however, they followed an unexpected pattern. It may be the results were partly due to error in measurement as blood pressure was only measured twice in the same setting on a single occasion. However, correlations of these measures between the first and second wave of measurement 4 years later were greater than 0.5 which suggests that substantial, stable variance was indexed by these measures. Blood pressure medication and higher systolic measures were associated with an increased risk, whereas higher diastolic measures were associated with a decreased risk of any-MCD. These findings partly contradict previous findings showing (1) a decreased risk of cognitive decline [35] and a milder occurrence of Alzheimer pathology [36] in individuals taking anti-hypertensive medication, and (2) an increased risk of developing dementia in hypertensive individuals [37], and should be interpreted cautiously. However, a Cochrane review [38] found no evidence of association between blood pressure-lowering treatments and the risk of developing dementia or cognitive impairment and we have not found studies reporting a risk associated with heightened diastolic blood pressure when other relevant variables were controlled. In addition, in the oldest-old, 
Table 4. Health and lifestyle predictors of conversion to any-MCD (full and reduced models)

\begin{tabular}{|c|c|c|c|c|}
\hline \multirow[t]{2}{*}{ Models } & \multicolumn{2}{|l|}{ Full } & \multicolumn{2}{|c|}{ Reduced } \\
\hline & Sig. & $\operatorname{Exp}(B)$ & Sig. & $\operatorname{Exp}(B)$ \\
\hline Systolic blood pressure, mm Hg & 0.154 & $1.01(0.99-1.03)$ & 0.057 & $1.02(1.00-1.04)$ \\
\hline Diastolic blood pressure, $\mathrm{mm} \mathrm{Hg}$ & 0.022 & $0.96(0.93-0.99)$ & 0.011 & $0.96(0.92-0.99)$ \\
\hline Arthritis & 0.253 & $1.39(0.79-2.44)$ & & \\
\hline FVC, 1 & 0.484 & $0.86(0.56-1.31)$ & & \\
\hline Diabetes & 0.842 & $1.09(0.48-2.44)$ & & \\
\hline BMI & 0.255 & $1.03(0.98-1.07)$ & 0.054 & $1.04(1.00-1.08)$ \\
\hline Physical health self-report & 0.892 & $1(0.97-1.03)$ & & \\
\hline Mental health self-report & 0.380 & $1.02(0.97-1.07)$ & & \\
\hline Visual acuity (Snellen) & 0.189 & $0.96(0.91-1.02)$ & & \\
\hline Taking blood pressure medication & 0.423 & $1.26(0.72-2.2)$ & & \\
\hline Taking anxiety medication & 0.526 & $0.67(0.2-2.28)$ & & \\
\hline Taking depression medication & 0.010 & $3.66(1.36-9.81)$ & 0.003 & $3.25(1.51-7.00)$ \\
\hline Taking cholesterol-lowering medication & 0.208 & $1.45(0.81-2.57)$ & & \\
\hline Goldberg anxiety scale & 0.416 & $0.93(0.79-1.1)$ & & \\
\hline Goldberg depression scale & 0.126 & $1.16(0.96-1.4)$ & & \\
\hline Past smoker & 0.023 & $1.94(1.1-3.42)$ & 0.018 & $1.97(1.12-3.44)$ \\
\hline Past alcohol intake category & 0.080 & $0.77(0.58-1.03)$ & 0.046 & $0.75(0.57-1.00)$ \\
\hline Past alcohol intake category (quadratic) & 0.166 & $1.14(0.95-1.37)$ & 0.087 & $1.17(0.98-1.40)$ \\
\hline $\mathrm{APOE}^{*} \mathrm{E} 4$ & 0.191 & $0.64(0.33-1.24)$ & & \\
\hline $\mathrm{APOE}^{*} \mathrm{E} 2$ & 0.272 & $1.47(0.74-2.92)$ & & \\
\hline \multirow[t]{2}{*}{ Model fit } & $\mathrm{R}^{2}$ & -2 Log likelihood & $\mathrm{R}^{2}$ & -2 Log likelihood \\
\hline & 0.105 & 518.55 & 0.075 & 533.66 \\
\hline
\end{tabular}

All models are adjusted for age, sex, and education.

it was found that higher systolic blood pressure was associated with better cognition [39] and in another longitudinal study investigating the relationship between blood pressure and late-life cognitive change, no linear association was found between systolic or diastolic blood pressure and cognition but a curvilinear relationship between diastolic blood pressure and cognition was present, showing an association between both high and low diastolic blood pressure and decreased cognition [40]. It may be that blood pressure medication and/or lower diastolic blood pressure in some individuals lead to chronic cerebral hypoperfusion/hypoxia [41] which over time might lead to neurodegeneration and negatively impact on cognition. These associations should be investigated further in future studies since, if they can be replicated, interventions focusing on low as well as high blood pressure management might be initiated to decrease the risk of cognitive decline in hypertensive individuals.

BMI, cholesterol-lowering medication, systolic blood pressure, diabetes, and diabetes medication were all found to be predictors of any-MCD. These findings are consistent with past research showing an association between the metabolic syndrome, cardiovascular disease, cognitive impairment, and dementia. However, while cognitive impairment was associated with greater BMI, higher systolic blood pressure, and having diabetes as expected, it was somewhat surprisingly positively associated with taking cholesterol-lowering medication and diabetes medication. Because these medications reduce the level of known risk factors (hypercholesterolemia and hyperglycemia), they could have been expected to be associated with decreased rather than increased cognitive impairment. In one clinical trial assessing anti-inflammatory drugs, participants who elected to use cholesterol medication had a lower risk of developing AD [42]. However, in large prospective epidemiological studies, cholesterol-lowering drugs were not found to be protective or a risk for $\mathrm{AD}$ [43]. The present findings are therefore likely to indicate that those individuals prescribed cholesterollowering medication are at higher risk due to other factors such as the pathological processes associated with high blood cholesterol prior to treatment. It may also be 
that if the cholesterol-lowering medication has been instituted relatively recently, it serves as a marker of disease without having had time to affect the course of neuropathological processes. It would therefore be preferable if information on the length of treatment be collected and included in the analyses of future studies of this kind.

Some variables emerged as potential predictors of conversion in univariate analyses but did not remain significant in multivariate analyses. They should be considered exploratory, and further follow-up is required to reveal whether their significance is reliable. For MCI, increased FVC, which may act as a proxy of physical and cardiovascular fitness, was associated with decreased risk of MCI and has been associated with better cognitive performance [44] and fewer white matter hyperintensities in the same study.

A previous study [3] investigating the predictors of $\mathrm{MCI}$ and $\mathrm{AD}$ in a larger and older cohort with substantially greater MCI prevalence found sex differences that were investigated in a stratified analysis. The number of converters in the present study did not provide a sufficient statistical power for such an analysis, but, although less sensitive, none of the sex interactions reached significance in the present study.

This study had a number of limitations. Beside those discussed above, many of the measures were based on self-report and are therefore open to bias. The FVC was based on 2 successive measures during the same session and may not be very reliable, although, as for the blood pressure measurements, correlations between the measures used in the present study and those collected in the second wave of measurement were high $(r>0.8)$. A relatively small number of participants converted to MCI and therefore some of the cells used for analysis were small. However, a number of risk factors for MCI were shared with any-MCD, which affected more participants, sug- gesting that the present findings are unlikely to be artefacts. It is also possible that some of these findings are underestimates, since the studied individuals were located in an affluent, well-educated city and probably engage in a healthier lifestyle with better dietary habits and lower smoking rates than the rest of the population $56 \%$ of participants ever smoked compared with $60 \%$ in the Australian population). Finally, despite the significance of these results in the assessment of population-based risks, they do not explain a sufficiently large proportion of the variance in clinical status to enable the classification of single individuals. Despite these limitations, theoretically relevant risk and protective factors were identified.

These findings indicate that lifestyle factors, and specifically smoking and alcohol consumption, are particularly important predictors of cognitive impairments in ageing populations and should be the focus of renewed interventions. These results also have clinical relevance, since as found in $\mathrm{AD}$ [30], anxiety and depression symptoms appear to be associated with a substantially greater risk of MCI. Finally, the possibility that blood pressure medications and low diastolic blood pressure might be associated with a greater risk of cognitive impairment should be further investigated.

\section{Acknowledgements}

The authors are grateful to Anthony Jorm, Bryan Rodgers, Karen Maxwell, June Cullen the Neuroimaging Group, NPI, Prince of Wales Hospital, and the PATH interviewers. The study was supported by NHMRC of Australia Unit Grant No. 973302, Program Grant No. 179805, NHMRC project grant No. 157125, grants from the Australian Rotary Health Research Fund and the Australian Brewers Foundation. N.C. is funded by Alzheimer's Australia Research and the Centre for Mental Health Research at the Australian National University. K.A. is funded by NHMRC Research Fellowship No. 366756.

\section{References}

1 Petersen RC, Negash S: Mild cognitive impairment: an overview. CNS Spectr 2008; 13 : 45-53.

-2 Mitchell AJ, Shiri-Feshki M: Rate of progression of mild cognitive impairment to dementia-meta-analysis of 41 robust inception cohort studies. Acta Psychiatr Scand 2009;119: 252-265.

- 3 Artero S, Ancelin ML, Portet F, et al: Risk profiles for mild cognitive impairment and progression to dementia are gender specific. J Neurol Neurosurg Psychiatry 2008;79:979984.

\footnotetext{
-4 Braak H, Alafuzoff I, Arzberger T, Kretzschmar H, Del Tredici K: Staging of Alzheimer disease-associated neurofibrillary pathology using paraffin sections and immunocytochemistry. Acta Neuropathol (Berl) 2006;112:389-404.

5 Skoog I, Gustafson D: Update on hypertension and Alzheimer's disease. Neurol Res 2006;28:605-611.

-6 Craft S: Insulin resistance and Alzheimer's disease pathogenesis: potential mechanisms and implications for treatment. Curr Alzheimer Res 2007;4:147-152.
}

\footnotetext{
7 Eggermont L, Swaab D, Luiten P, Scherder E: Exercise, cognition and Alzheimer's disease: more is not necessarily better. Neurosci Biobehav Rev 2006;30:562-575.

-8 Van Den Heuvel C, Thornton E, Vink R: Traumatic brain injury and Alzheimer's disease: a review. Prog Brain Res 2007;161:303316.

-9 Anstey KJ, Lipnicki DM, Low LF: Cholester$\mathrm{ol}$ as a risk factor for dementia and cognitive decline: a systematic review of prospective studies with meta-analysis. Am J Geriatr Psychiatry 2008;16:343-354.
} 
10 O’Keefe JH, Bybee KA, Lavie CJ: Alcohol and cardiovascular health: the razor-sharp double-edged sword. J Am Coll Cardiol 2007;50: 1009-1014.

-11 Thomas AJ, O’Brien JT: Depression and cognition in older adults. Curr Opin Psychiatry 2008;21:8-13.

-12 AnsteyKJ, vonSanden C, Salim A, O’Kearney $\mathrm{R}$ : Smoking as a risk factor for dementia and cognitive decline: a meta-analysis of prospective studies. Am J Epidemiol 2007;166: 367-378.

13 Jackson GR, Owsley C: Visual dysfunction, neurodegenerative diseases, and aging. Neurol Clin 2003;21:709-728.

14 Ertekin-Taner N: Genetics of Alzheimer's disease: a centennial review. Neurol Clin 2007;25:611-667.

15 Ritchie K, Artero S, Touchon J: Classification criteria for mild cognitive impairment: a population-based validation study. Neurology 2001;56:37-42.

-16 Anstey KJ, Cherbuin N, Christensen H, et al: Follow-up of mild cognitive impairment and related disorders over 4 years in adults in their sixties: the PATH Through Life Study. Dement Geriatr Cogn Disord 2008;26:226233.

-17 Jorm AF, Anstey KJ, Christensen H, Rodgers B: Gender differences in cognitive abilities: the mediating role of health state and health habits. Intelligence 2004;32:7-23.

-18 Kumar R, Dear KB, Christensen H, et al: Prevalence of mild cognitive impairment in 60- to 64-year-old community-dwelling individuals: the Personality and Total Health Through Life 60+ Study. Dement Geriatr Cogn Disord 2005;19:67-74.

-19 Petersen RC, Smith GE, Waring SC, Ivnik RJ, Tangalos EG, Kokmen E: Mild cognitive impairment: clinical characterization and outcome. Arch Neurol 1999;56:303-308.

20 Kral VA: Senescent forgetfulness: benign and malignant. Can Med Assoc J 1962;86: 257-260.

21 Crook T, Bartus RT, Ferris SH, Whitehouse P, Cohen GD, Gershon S: Age-associated memory impairment: proposed diagnostic criteria and measures of clinical change. Report of a National Institute of Mental Health work group. Devel Neuropsychol 1986;2: 261-276.

22 American Psychiatric Association: Diagnostic and Statistical Manual of Mental Disorders (DSM-IV). Washington, APA, 1994.
23 Morris JC: The clinical dementia rating (CDR): current version and scoring rules. Neurology 1993;43:2412-2414.

24 Kumar R, Parslow RA, Jorm AF, et al: Clinical and neuroimaging correlates of mild cognitive impairment in a middle-aged community sample: the Personality and Total Health Through Life 60+ Study. Dement Geriatr Cogn Disord 2006;21:44-50.

25 Ware J Jr, Kosinski M, Keller SD: A 12-item Short-Form Health Survey: construction of scales and preliminary tests of reliability and validity. Med Care 1996;34:220-233.

26 Cherbuin N, Anstey KJ, Sachdev PS, et al: Total and regional gray matter volume is not related to $\mathrm{APOE}^{*} \mathrm{E} 4$ status in a community sample of middle-aged individuals. J Gerontol A Biol Sci Med Sci 2008;63:501-504.

27 Goldberg D, Bridges K, Duncan-Jones P, Grayson D: Detecting anxiety and depression in general medical settings. BMJ 1988; 297:897-899.

28 Chodosh J, Kado DM, Seeman TE, Karlamangla AS: Depressive symptoms as a predictor of cognitive decline: MacArthur Studies of Successful Aging. Am J Geriatr Psychiatry 2007;15:406-415.

29 Saunders JB, Aasland OG, Babor TF, de la Fuente JR, Grant M: Development of the Alcohol Use Disorders Identification Test (AUDIT): WHO Collaborative Project on Early Detection of Persons with Harmful Alcohol Consumption - II. Addiction 1993;88: 791-804.

30 Palmer K, Berger AK, Monastero R, Winblad B, Backman L, Fratiglioni L: Predictors of progression from mild cognitive impairment to Alzheimer disease. Neurology 2007; 68:1596-1602.

31 Schroeter MR, Sawalich M, Humboldt T, et al: Cigarette smoke exposure promotes arterial thrombosis and vessel remodeling after vascular injury in apolipoprotein E-deficient mice. J Vasc Res 2008;45:480-492.

32 Gache P, Michaud P, Landry U, et al: The Alcohol Use Disorders Identification Test (AUDIT) as a screening tool for excessive drinking in primary care: reliability and validity of a French version. Alcohol Clin Exp Res 2005;29:2001-2007.

33 Solfrizzi V, D’Introno A, Colacicco AM, et al: Alcohol consumption, mild cognitive impairment, and progression to dementia. Neurology 2007;68:1790-1799.
34 Rodgers B, Windsor TD, Anstey KJ, Dear $\mathrm{KB}$, A FJ, Christensen $\mathrm{H}$ : Non-linear relationships between cognitive function and alcohol consumption in young, middle-aged and older adults: the PATH Through Life Project. Addiction 2005;100:1280-1290.

35 Hanon O, Pequignot R, Seux ML, et al: Relationship between antihypertensive drug therapy and cognitive function in elderly hypertensive patients with memory complaints. J Hypertens 2006;24:2101-2107.

-36 Hoffman LB, Schmeidler J, Lesser GT, et al: Less Alzheimer disease neuropathology in medicated hypertensive than nonhypertensive persons. Neurology 2009; 72:1720-6.

$>37$ Reitz C, Tang MX, Manly J, Mayeux R, Luchsinger JA: Hypertension and the risk of mild cognitive impairment. Arch Neurol 2007;64:1734-1740.

38 McGuinness B, Todd S, Passmore P, Bullock $\mathrm{R}$ : The effects of blood pressure lowering on development of cognitive impairment and dementia in patients without apparent prior cerebrovascular disease. Cochrane Database Syst Rev 2006;CD004034.

39 Nilsson SE, Read S, Berg S, Johansson B, Melander A, Lindblad U: Low systolic blood pressure is associated with impaired cognitive function in the oldest old: longitudinal observations in a population-based sample 80 years and older. Aging Clin Exp Res 2007; 19:41-47.

40 Hebert LE, Scherr PA, Bennett DA, et al: Blood pressure and late-life cognitive function change: a biracial longitudinal population study. Neurology 2004;62:2021-2024.

41 Henry-Feugeas MC: Alzheimer's disease in late-life dementia: a minor toxic consequence of devastating cerebrovascular dysfunction. Med Hypotheses 2008;70:866875.

42 Sparks DL, Kryscio RJ, Sabbagh MN, Connor DJ, Sparks LM, Liebsack C: Reduced risk of incident $\mathrm{AD}$ with elective statin use in a clinical trial cohort. Curr Alzheimer Res 2008;5:416-421.

43 Arvanitakis Z, Schneider JA, Wilson RS, et al: Statins, incident Alzheimer disease, change in cognitive function, and neuropathology. Neurology 2008;70:1795-1802.

44 Anstey KJ, Windsor TD, Rodgers B, Jorm AF, Christensen $\mathrm{H}$ : Lower cognitive test scores observed in alcohol abstainers are associated with demographic, personality, and biological factors: the PATH Through Life Project. Addiction 2005;100:1291-1301. 